\title{
$\infty \sqrt{1}$ Stroke and \\ Therapeutic application of exosomes in ischaemic stroke
}

\author{
Yongfang Li, ${ }^{1}$ Yaohui Tang, ${ }^{2}$ Guo-Yuan Yang (D) ${ }^{1,2}$
}

To cite: Li Y, Tang Y, Yang G-Y. Therapeutic application of exosomes in ischaemic stroke. Stroke \& Vascular Neurology 2021;6: e000419. doi:10.1136/ svn-2020-000419

Received 5 May 2020 Revised 28 August 2020 Accepted 18 September 2020 Published Online First 11 January 2021
Check for updates

(C) Author(s) (or their employer(s)) 2021. Re-use permitted under CC BY-NC. No commercial re-use. See rights and permissions. Published by BMJ.

${ }^{1}$ Department of Neurology, Ruijin Hospital, School of medcine,

Shanghai Jiao Tong University,

Shanghai, China

${ }^{2}$ Neuroscience and Neuroengineering Center, Medx Research Institute, Shanghai Jiao Tong University School of Biomedical Engineering, Shanghai, China

Correspondence to Dr Guo-Yuan Yang; gyyang@sjtu.edu.cn

\section{ABSTRACT}

Ischaemic stroke is a leading cause of long-term disability in the world, with limited effective treatments. Increasing evidence demonstrates that exosomes are involved in ischaemic pathology and exhibit restorative therapeutic effects by mediating cell-cell communication. The potential of exosome therapy for ischaemic stroke has been actively investigated in the past decade. In this review, we mainly discuss the current knowledge of therapeutic applications of exosomes from different cell types, different exosomal administration routes, and current advances of exosome tracking and targeting in ischaemic stroke. We also briefly summarised the pathology of ischaemic stroke, exosome biogenesis, exosome profile changes after stroke as well as registered clinical trials of exosome-based therapy.

\section{INTRODUCTION}

Ischaemic stroke, accounting for more than $80 \%$ of stroke population, is a severe worldwide disease with high disability and heavy economic burdens. ${ }^{12}$ However, the clinical treatments are limited with only recombinant tissue plasminogen activator and thrombectomy in the very acute phase. ${ }^{3}$ Therefore, it is urgent and crucial to develop feasible therapeutic treatments in stroke.

Recently, exosomes have gained increasing interest in stroke due to their diagnostic and paramount therapeutic applications. ${ }^{4-6}$ Exosomes are a subset of extracellular vesicles with a diameter ranging from 30 to $150 \mathrm{~nm},{ }^{7}$ and their regenerative roles in stroke have been well documented. ${ }^{468}$ Compared with cell-based therapy, exosome-based therapy shows similar substantial protective effects while reduces potential tumourigenic and immunogenic side effects. ${ }^{4}$ The underlying mechanisms of exosome therapeutic effects are primarily via transferring their enriched cargo, especially microRNAs (miRNAs). Mature miRNAs are single-stranded nucleic acid consisting of 20-25 nucleotides and can target multiple genes via binding to the 3'-untranslated region and repressing mRNA transcription, which can be specifically enriched in exosomes and regulate the proliferation, apoptosis, survival and differentiation of target cells. ${ }^{910}$ These mechanisms have been carefully discussed in previous reviews. ${ }^{468}$
Herein, we mainly focus on current advances in the therapeutic application of different cell derived exosomes, exosome administration routes, exosome tracking and targeting in ischaemic stroke. We also briefly discuss the pathology of ischaemic stroke, biogenesis of exosomes, exosome profile changes after stroke and registered exosome-based clinical trials for stroke.

\section{ISCHAEMIC STROKE PATHOLOGY}

Ischaemic stroke could immediately result in ion balance disruption, metabolism failure and reduce general protein synthesis in brain cells, then induce peri-infarct depolarisation, excitotoxicity, oxidative stress, inflammatory response and blood-brain barrier (BBB) disruption, which altogether cause the death of neurons, astrocytes, microglia, oligodendrocytes and endothelia cells. ${ }^{11} 12$ Noteworthily, cells in the peri-infract area are still alive in the early phase of ischaemia; however, most of them are difficult to survive due to the unfriendly microenvironment. It is important to note that with these progressions, stroke also initiates the repair and remodelling processes soon after, including angiogenesis, neurogenesis, synaptogenesis and oligodendrogenesis. ${ }^{113}$ Finally, newly formed neurovascular unit could partially replace the injured tissue and improve outcomes. ${ }^{14}$

\section{Inflammation response after ischaemic stroke}

Inflammatory response possesses both the detrimental and beneficial influences in the progression of cerebral ischaemia. ${ }^{15-17}$ In the acute phase of stroke, inflammation could over-activate immune system. Resident microglia and infiltrating peripheral immune cells are activated by inflammatory factors or cellular components released from dying cells, then producing various inflammatory factors, chemokines, adhesion molecules and tissuedestructive enzymes, and activating complement system. ${ }^{18-20}$ These altogether accelerate BBB damage and exacerbate brain injury in a vicious circle. ${ }^{19}$ On the other hand, immune cells could also release anti-inflammatory factors and neurotrophic factors, clear cell 
debris and toxic substances and promote neurovascular unit repair, especially in the late phase. ${ }^{1621}$ The detailed inflammatory and immune responses after stroke are discussed in these reviews. ${ }^{16-19} 2122$

\section{BBB disruption and recovery after ischaemic stroke}

BBB is structurally formed by endothelia cells, pericytes, base membrane, astrocyte endfeet, the linking tight and gap junctions and extracellular matrix components. ${ }^{23}$ BBB dynamically mediates substance exchange and cellular crosstalk between brain and peripheral blood, and maintains cerebral microenvironment homeostasis. ${ }^{24}$ Following ischaemic stroke, dysfunctions of endothelia cells, pericytes and astrocytes, disruptions of tight junctions and basement membrane, oxidative stress and inflammatory response altogether lead to increasing BBB permeability, and even cause its breakdown. ${ }^{23}{ }^{25-27}$ BBB disruption could induce vasogenic oedema, extravasation of toxic blood components and infiltration of immune cells in brain parenchyma. Consequently, it causes secondary brain injury. ${ }^{28}$ The BBB disruption usually lasts for 7 days after ischaemic stroke. After then, BBB permeability recovers and gradually returns to normal state due to the decrease of inflammatory factors, increase of growth factors, proregenerative effects of immune cells and integration of progenitor cells. ${ }^{28}$

\section{Angiogenesis, neurogenesis and oligodendrogenesis after ischaemic stroke}

Following ischaemia onset, endothelial cells, neurons, astrocytes, microglia and oligodendrocytes mainly undergo necrosis in the ischaemic core region. ${ }^{11} 29$ Though the border zone surrounding the core, known as ischaemic penumbra, is less affected and has collateral blood supply, cells in this area are challenged with autophagy and apoptotic cell death. ${ }^{30}{ }^{31}$ For the detailed mechanisms of cell death and oxidative stress after stroke, readers can refer to these reviews. ${ }^{32-38}$ Meanwhile, brain ischaemia also triggers local angiogenesis, neurogenesis and oligodendrogenesis.

Angiogenesis involves the coordination of basal lamina matrix detachment and remodelling, endothelial cell proliferation, migration and elongation, along with the participation of pericytes to form new microvessels. ${ }^{39}$ Stroke induces the overexpression of matrix metalloproteases, which are important for basal lamina matrix detachment. ${ }^{40} 41$ Moreover, ischaemia upregulates integrins and angiogenic factors including vascular endothelial growth factor (VEGF), platelet-derived growth factor, fibroblast growth factor (FGF), angiopoietin-1/-2 and Tie-2, which could activate endothelial cells and promote angiogenesis. ${ }^{39}$ In addition, endothelial progenitor cells are motivated and participated in angiogenesis. ${ }^{42}{ }^{43}$ Adult neural stem cells (NSCs) are found to located in the subventricular zone of lateral ventricle, the subgranular zone of dentate gyrus and the posterior periventricular area. ${ }^{445}$ Ischaemia could induce the expression of neurogenic factors such as epidermal growth factor receptor
(EGFR), transforming growth factor $\alpha$ (TGF- $\alpha$ ), FGF, chemokine such as stromal cell-derived factor 1 and transcription factors, which accompanies with the repair of injured neuronal axon, dendrites and synapses. ${ }^{446}$ These bioactive molecules can activate NSC proliferation, migration along white matter fibre tracts or blood vessels ${ }^{47}$ and differentiation. Recently, reactive astrocytes were found to trans-differentiate into neurons after ischaemia, ${ }^{48}$ and they had great potentials to be reprogrammed into neuron via overexpression of neurogenic transcription factors including NeuroD1, Sox2, neurogenin- $2 .{ }^{49}{ }^{50}$ The proliferation, migration and maturation of oligodendrocyte progenitor cells contribute to oligodendrogenesis and remyelination after cerebral ischaemia. ${ }^{51}$ However, the differentiation of oligodendrocyte progenitor cells into myelinating oligodendrocytes is limited and impaired in the injured brain. ${ }^{52}$ Therefore, therapeutic strategies aiming at modulating and enhancing endogenous repair process exert promising potential in ischaemic stroke treatment.

\section{BIOGENESIS OF EXOSOMES}

Exosomes are special extracellular vesicles originated from endosomes with a size of $30-150 \mathrm{~nm}$ in diameter. ${ }^{5354}$ They were first found in investigating the transferrin receptor fate during the maturation of sheep reticulocytes into red blood cells in $1983,{ }^{55} 56$ and named as 'exosomes' in $1987 .{ }^{54}$ Since then, successive studies demonstrated that various types of cells were able to release exosomes, from immune cells, tumour cells, epithelial cells to neurons and glia cells. The presence of exosomes were also discovered in all kinds of biofluids including blood, urine, saliva, cerebrospinal fluid, bile, semen, ascites, amniotic fluid and breastmilk. ${ }^{53}{ }^{57}$ In late 1990s, exosomes were found to function as intercellular communication mediators. ${ }^{58} 59$ Excitingly, exosomes were first found to carry nucleic acids such as miRNA and mRNA in $2007 .{ }^{60}$ Now, it is well accepted that exosomes contain certain enriched proteins, lipids, DNAs, mRNAs and miRNAs derived from their original cells, and can be released by cells with active biological functions. ${ }^{5761}$

The biogenesis of exosomes involves the formation of multivesicular bodies (MVBs) from early endosomes, the produce of intraluminal vesicles (ILVs) in MVBs as well as the transport and fusion with plasm membrane to release ILVs from MVBs. ${ }^{57}$ Inward budding of early endosome membrane forms MVBs and then further ILVs. The endosomal sorting complexes required for transport with their associated proteins (ALIX, VPS4, etc), tetraspanin proteins (CD63, CD81, etc) and chaperone proteins (HSP70) are involved in cargo sorting. ${ }^{57} 61$ Then, the Rab proteins and other unknown intracellular effectors modulate the transportation and secretion of MVBs. For detailed descriptions of exosome formation and secretion, please referred to these reviews. ${ }^{53561}$

Exosomes are small and non-toxic extracellular vesicles, which can easily cross the BBB without provoking immune 
responses as a novel shuttle. ${ }^{62}$ These properties make exosomes an ideal and adjunct therapeutic approach for post-injury regeneration, such as engineering exosomes as exquisite delivery platforms for drugs, bioactive molecules and specific immune-modulators. ${ }^{4} 6364$

\section{EXOSOME PROFILE CHANGES AFTER STROKE}

After stroke, the profile of exosomes synthesised and released from brain cells alters and these exosomes can pass through BBB to cerebrospinal fluid or peripheral blood, which can possibly serve as potential biomarkers for stroke diagnosis and prognosis. ${ }^{6365} 66$ Additionally, exosomes are also released to blood from endothelial and blood cells in response to stroke. Several studies have examined the changes of circulating exosomal contents including proteins and nucleic acids in stroke. In a proteome screening of microvesicles from five lacunar infarction patients' plasma and follow-up study of 5 years' outcomes, the upregulation of myelin basic protein, coagulation cascade proteins and focal adhesion, and the downregulation of albumin was associated with adverse outcomes. ${ }^{67}$ Another study examined inflammasome proteins form serum and serum exosomes in patients who had a stroke, results suggested that apoptosis-associated speck-like protein was a potential biomarker for stroke. ${ }^{68}$ Besides, exosomal proteins cystatin $\mathrm{C}$ and $\mathrm{CD} 14$ were related with cerebral white matter lesions and progressing brain atrophy. ${ }^{69}$

As for exosomal miRNA pattern changes, serum exosomal miR-126 was found to decrease at 3 hours and back to normal at 24 hours after rat transient and permanent ischaemia, while serum miR-126 showed no significant changes in transient ischaemia, suggesting that exosomal miR-126 may be more sensitive and specific to ischaemia. ${ }^{70}$ In patients with acute stroke, serum exosomal miR-9 and miR-124 were in significantly higher levels and positively associated with clinical scores. ${ }^{71}$ Similarly, analysing circulating exosomal from patients who had an ischaemic stroke within 24 hours or 72 hours onset found that miR-134 and miR-233, respectively were upregulated and positively correlated with severe clinical scores, poor outcomes and inflammatory responses. ${ }^{72} 73$ Moreover, plasma exosomal miR-422a and miR-125b-2-3 p were found to decrease in subacute phase of ischaemic stroke while miR-422a increased in the acute phase, and both could serve as biomarkers for ischaemic monitor and diagnosis. ${ }^{74}$ Detecting plasma exosomal miRNA-30a-5p and miR-21-5 p from 143 ischaemic patients divided into hyperacute, acute, subacute and recovery phases showed that combination of these two miRNAs can be used for diagnosing and distinguishing different phases of ischaemic stroke. ${ }^{75}$

Importantly, some treatments for stroke could also induce the change of exosomes. In mice transient ischaemic stroke, mice received moderate exercise for 4 weeks before ischaemia showed elevated miR-126 levels in endothelial progenitor cell exosomes isolated form the total brain tissue and periphery circulation exosomes. This miRNA was associated with neuron protection against apoptosis, axon growth, angiogenesis and neurogenesis. ${ }^{76}$ Similarly, in rat cardiac ischaemia, long term exercise elevated circulating exosomal miR-342-5 p levels and prevented cardiomyocyte from apoptosis via targeting caspase 9 and Jnk2. Further analysis of the origin of exosomal miR-342-5 $\mathrm{p}$ showed that exercise directly promoted endothelial cell to synthesis miR-342-5p. ${ }^{77}$ In semaphorin 3A (Sema-3A, a molecule that inhibits axonal outgrowth) inhibitor treatment of rat ischaemic stroke, Sema-3A inhibitor induced the upregulation of several genes including ptgds in astrocyte-derived exosomes. ${ }^{78}$

\section{EXOSOME-BASED THERAPY AND APPLICATION \\ Mesenchymal stem cell derived exosomes}

Mesenchymal stem cell (MSC) transplantation has been shown to promote post-stroke recovery in animal models and clinical trials (table 1) ${ }^{79}$ Growing evidence suggests that stem cells exert their therapeutic effects mainly via paracrine mechanisms, among which exosome releasing is intensively investigated. ${ }^{46}$ In ischaemia, MSC derived exosomes have been well shown to promote stroke recovery.

In rat transient middle cerebral artery occlusion (tMCAO) model, intravenously delivered MSC exosomes enhanced function recovery via promoting neurogenesis, neurite remodelling and angiogenesis. ${ }^{80}$ In order to compare the therapeutic effects of MSC exosomes and MSCs in ischaemia, MSC exosomes were intravenously administrated 1, 3 and 5 days after tMCAO, while MSCs were given 1 day after tMCAO. Results showed that MSC exosomes exhibited closely the same effects as MSCs in terms of neuroprotection, angiogenesis and immunomodulation. ${ }^{81}{ }^{82}$ Interestingly, the effects of MSC exosomes partly depend on MSC culturing conditions. For instance, exosomes derived from brain extract treated or oxygen glucose deprivation (OGD) treated MSCs showed better therapeutic effects, which could be due to the enrichment of some certain functional proteins in exosomes. ${ }^{8384}$ Excitingly, MSC exosomes were also proved to be effective in primate stroke model. In macaca mulatta cortical hand motor cerebral injury model, intravenous injection of MSC exosomes 24 hours and 14 days post-injury promoted fine hand motor function recovery. ${ }^{66}$ Further mechanism studies reveal that MSC exosomes not only reduced neuroinflammation and shift microglia into restorative functions in aged macaca mulatta, ${ }^{85}$ but also hampered injury related hyperexcitability and restored excitatory-inhibitory balance. ${ }^{86}$

These effects could be ascribed to functional miRNAs or proteins transferring and further downstream signalling pathway activation. Intranasal delivery of MSC exosomes before ischaemia reduced neuronal death, promoted oligodendroglia maturation and inhibited microglia-mediated neuro-inflammation possibly via Toll-like receptor 4/CD14/NF-kB signalling pathway. ${ }^{87} 88$ 


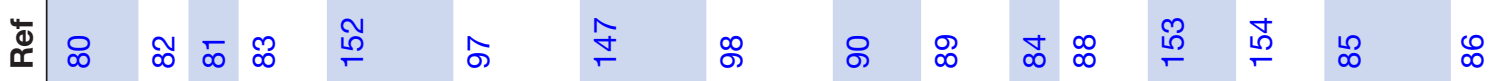
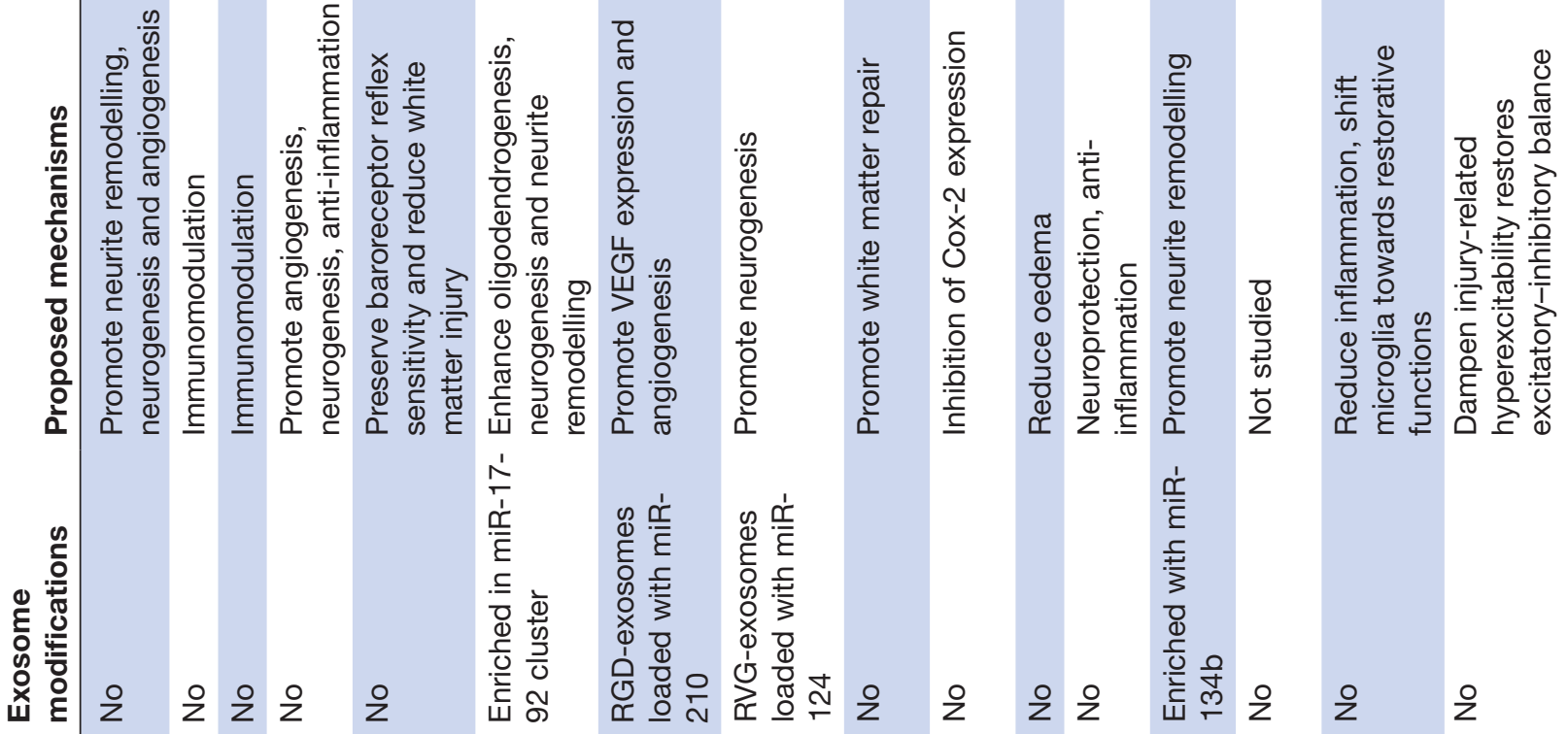

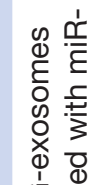

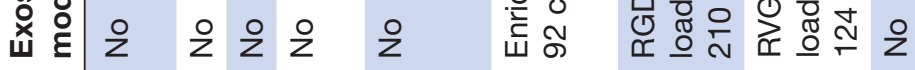

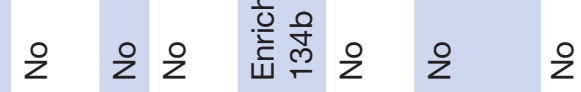
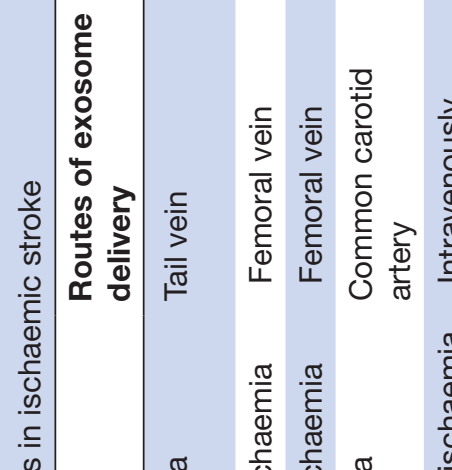

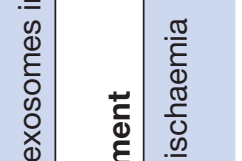

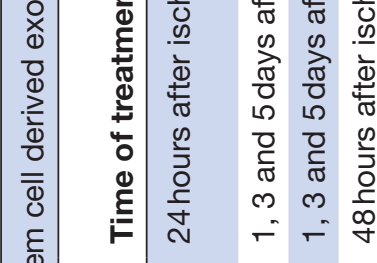

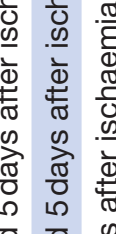

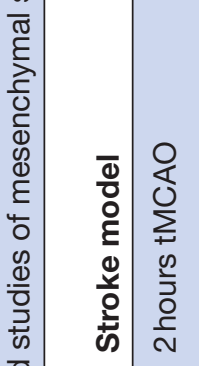<smiles>CCC</smiles>

$\frac{\dot{\omega}}{x}$

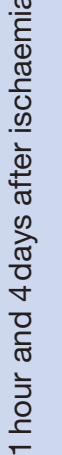

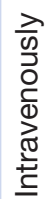
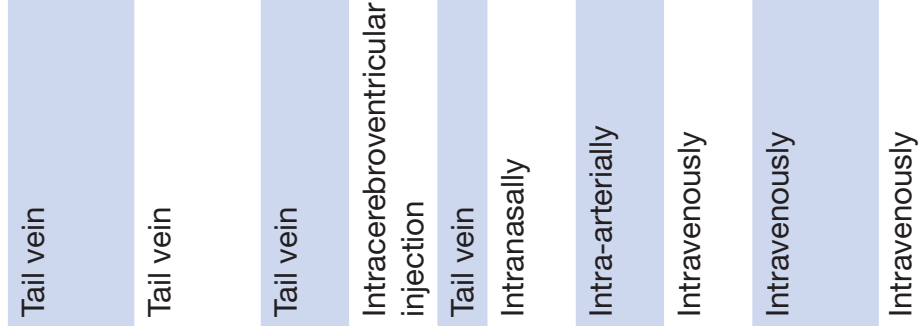

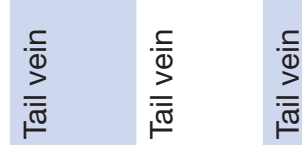

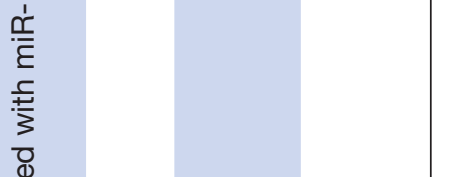


In global ischaemia, MSC exosomes ameliorated hippocampal spatial learning and memory deficits potentially through inhibition of cyclo-oxygenase- 2 expression. ${ }^{89}$ In subcortical ischaemia, MSC exosomes promoted axonal sprouting and white matter repair. ${ }^{90}$ The promotion of axonal growth was mediated by exosomal transferring miR-17-92 cluster and activating the PTEN/mTOR signal pathway in vitro. ${ }^{91}$ The protection of oligodendrocyte from apoptosis was through targeting caspase- 8 by exosomal miR-134. ${ }^{92}$ Another study using lentiviruses to knock-in and knock-down miR-133b in MSCs showed that the effects of MSCs was partially mediated by exosomal transferring miR-133b to the astrocytes and neurons. ${ }^{93}$ In OGD, MSC exosomes protected neurons from apoptosis via transferring let-7-5 p and subsequently inhibiting caspase-3 expression. ${ }^{94}$

Recently, strategies of engineering exosomes to enhance their therapeutic effects are emerging as hotspots. ${ }^{95}{ }^{96}$ For instance, MSC exosomes enriched with the miR-17-92 cluster showed greater improvements on oligodendrogenesis, neurogenesis and neurite remodelling than control. ${ }^{97}$ In a mouse photothrombosis model, researchers modified MSC exosomes with rabies virus glycoprotein (RVG, a neuron targeting peptide) by fusing the peptide with exosomal protein lysosome-associated membrane glycoprotein 2b (Lamp2b), then loaded with miR-124-mimics via electroporation. These engineered exosomes were shown to efficiently carry miRNA-124 to the ischaemic region and ameliorate brain injury by promoting neural progenitor differentiation. ${ }^{98}$

\section{NSC derived exosomes}

The investigations of NSC derived exosomes in stroke have been attracting interest since 2018 (table 2). A study compared the effect of exosomes from NSCs and MSCs, which were derived from the same pluripotent stem cell line in thromboembolic stroke model. NSC exosomes were proved to exert greater function improvement and infarct volume reduce than MSC exosomes, which were associated with more potent effect in polarising macrophage toward M2 phenotype and inhibiting inflammation. ${ }^{99}$ Additionally, the effect of NSC exosomes on reducing infarct volume may through preservation of astrocyte function. ${ }^{100}$ Moreover, NSC exosomes also showed promising therapeutic effects in aged stroke mice. ${ }^{99}$ Importantly, in porcine stroke model, NSC exosome treatment also reduced infarct volume and brain oedema, promoted white matter integrity and function recovery. ${ }^{101}$

\section{Adipose-derived stem cells derived exosomes}

Adipose-derived stem cells (ADSCs) can be easily obtained from surgical adipose waste tissue and have been proven to be a promising candidate for ischaemic treatment. Recently, exosomes from ADSCs are emerging as a substitutive therapy (table 3). ${ }^{102}$ In vitro, ADSC exosomes promoted brain microvessel endothelial cell migration and tube formation, while tailored miR-181b-5p overexpressing ADSC exosomes further enhanced angiogenesis via downregulating its target transient receptor potential melastatin 7 and upregulating hypoxia-inducible factor- $1 \alpha$ and VEGF. ${ }^{103}$ Systemic administration of miRNA-126 overexpressed ADSC exosomes could effectively inhibit neuro-inflammation, reduce neuron death, promote neurogenesis and functional recovery than normal and miRNA-126 knockdown ADSC exosomes in rat ischaemia. ${ }^{104}$ Similarly, ADSC exosomes enriched with miR-30d-5p further prevented cerebral injury via promoting M2 microglia polarisation than normal and miR-30d-5p knock-down group. Mechanism study showed that miR-30d-5p suppressed autophagy-mediated M1 microglia polarisation by targeting autophagy-related genes beclin-1 and autophagy-related genes $5 .{ }^{105} 109 \mathrm{In}$ rat ischaemia, intraventricular administration of ADSC exosomes engineered with a multifunction protein pigment epithelium-derived factor more potentially repressed neuron apoptosis via promoting autophagyassociated protein little computer 3 expression. ${ }^{106}$

\section{Other cell derived exosomes}

Apart from stem cells, other cells derived exosomes also exhibited therapeutic effects in stroke (table 4). Here, we discussed the applications of exosomes from astrocytes, endothelial cells and microglia.

Astrocyte derived exosomes were showed to reduce infarct volume and inhibit neuron apoptosis via

Table 2 Published studies of neural stem cell derived exosomes in ischaemic stroke

\begin{tabular}{|c|c|c|c|c|c|c|}
\hline Animals & Stroke model & Time of treatment & $\begin{array}{l}\text { Routes of } \\
\text { exosome delivery }\end{array}$ & $\begin{array}{l}\text { Exosome } \\
\text { modifications }\end{array}$ & Proposed mechanisms & Ref \\
\hline Mouse & TE-MCAO & $\begin{array}{l}2 \text { hours, } 14 \text { hours, } \\
38 \text { hours/ } 6 \text { hours, } 24 \text { hours, } \\
48 \text { hours (in aged mice) } \\
\text { after ischaemia }\end{array}$ & Tail vein & No & $\begin{array}{l}\text { Immunomodulation, inhibit } \\
\text { inflammation }\end{array}$ & 99 \\
\hline Mouse & 1 hour tMCAO & 2 hours after ischaemia & $\begin{array}{l}\text { Internal jugular } \\
\text { vein }\end{array}$ & No & Preserve astrocyte function & 100 \\
\hline
\end{tabular}

BBB, blood-brain barrier; pMCAO, permanent middle cerebral artery occlusion; TE-MCAO, thromboembolic middle cerebral artery occlusion; WM, white matter. 


\begin{tabular}{|c|c|c|c|c|c|c|}
\hline Animals & $\begin{array}{l}\text { Stroke } \\
\text { model }\end{array}$ & $\begin{array}{l}\text { Time of } \\
\text { treatment }\end{array}$ & $\begin{array}{l}\text { Routes of } \\
\text { exosome delivery }\end{array}$ & Exosome modifications & Proposed mechanisms & Ref \\
\hline Rat & tMCAO & $\begin{array}{l}\text { Immediately after } \\
\text { ischaemia }\end{array}$ & Tail vein & $\begin{array}{l}\text { Enriched with miR-30d- } \\
5 p\end{array}$ & $\begin{array}{l}\text { Reduce autophagy and } \\
\text { inflammation, and promote } \\
\text { microglia M2 polarisation }\end{array}$ & 105 \\
\hline Rat & tMCAO & Not mentioned & Intravenously & Enriched with miR-126 & $\begin{array}{l}\text { Promote neurogenesis, } \\
\text { angiogenesis, anti- } \\
\text { inflammation }\end{array}$ & 104 \\
\hline Rat & $\begin{array}{l}1 \text { hour } \\
\text { tMCAO }\end{array}$ & $\begin{array}{l}3 \text { days before } \\
\text { ischaemia }\end{array}$ & $\begin{array}{l}\text { Lateral cerebral } \\
\text { ventricle injection }\end{array}$ & $\begin{array}{l}\text { Loaded with pigment } \\
\text { epithelium-derived factor }\end{array}$ & Promote autophagy & 156 \\
\hline
\end{tabular}

tMCAO, transient middle cerebral artery occlusion.

regulating autophagy. ${ }^{107}$ Exosomes from OGD preconditioned astrocytes attenuated OGD induced neuron death via shedding miR-92b-3p. ${ }^{108}$ In rat permanent ischaemia, Sema-3A inhibitor could reduce astrocyte activation and promote axonal elongation via GTPase-1/R-Ras/Akt/ GSK-3 $\beta$ signalling pathway. They also confirmed that exosomes from OGD Sema-3A inhibitor treated astrocyte further promoted neuron axonal outgrowth than exosomes from normal and OGD astrocyte. ${ }^{78}$

However, these effects were adverse in normal and OGD endothelial cells exosomes. Exosomes from normal microvascular endothelial cells inhibited astrocyte apoptosis in vitro, ameliorated BBB disruption, reduced infarct volume and promoted neurological function recovery in vivo, while OGD endothelial cell exosomes all presented reversed effects. ${ }^{109}$ Differences in exosomal protein and miRNA contents isolated from the same cell under different cultures may explain for these discrepancies. ${ }^{110}$ Interestingly, remote ischaemic postconditioning increased exosomes in serum and reduced ischaemia-reperfusion injury partially via exosomes released from endothelial cells in femoral arteries. ${ }^{111} 112$ Treating SH-SY5Y cells with exosomes from OGD human umbilical vein endothelial cells (HUVEC) inhibited cell apoptosis, promoted cell migration and invasion under OGD. ${ }^{112}$ Moreover, endothelial cell exosomes suppressed macrophage activation and inflammation via transferring miR-10a and inhibiting NF-kB signalling pathway. ${ }^{113}$ Intravenously injecting brain endothelial cell exosomes 3 days after ischaemia in diabetes mice improved neurological outcomes and cognitive function, enhanced axon outgrowth and myelin density, promoted angiogenesis and M2 macrophage polarisation. However, miR-126 knockdown endothelial cell exosomes largely abolished these beneficial effects. ${ }^{114}$

Exosomes from IL-4 stimulated BV2 microglia cells promoted HUVEC tube formation. ${ }^{115}$ Systemic administration of M2 microglial exosomes reduced infarct volume, promoted neurological function recovery and inhibited neuron apoptosis possibly via transferring miR-124 and modulating ubiquitin-specific protease 14 in neurons. ${ }^{116}$ These studies collectively suggested that exosomes play a critical role in intercellular communication and could be used for therapeutic applications.

\section{ROUTES OF EXOSOME ADMINISTRATION}

There are different exosome administration routes in stroke, which could be divided into two broad categories, respectively, systemic and local administration. Systemic administration includes intravenous injection via tail vein, femoral vein or internal jugular vein, intra-arterial injection via common carotid artery, intraperitoneal and intranasal delivery. Exosomes delivered via tail vein is the most used route in rodent models of ischaemic stroke. ${ }^{80} 909899105109116$ Visualisation of indium111-labelled exosomes injected 1-hour post ischaemia by single photon emission CT (SPECT) showed that exosomes appeared at the infarcted area 1-hour post injection, and were largely cleared from the brain by 24 hours. $^{99}$ Another study revealed that the clear time of exosomes from blood rapidly began 1 hour and gradually lasted from 1.5 to 6 hours post injection. ${ }^{117}$ Similarly, $3{ }^{125}$ I-iodobenzoyl norbiotinamide labelled exosomes displayed a distribution phase with a halflife of $1.5 \mathrm{~min}$ then an elimination phase with a longer half-life of 6 hours via measuring the radioactivity in the blood. ${ }^{118}$ Notably, the majority of exosomes were trapped in the liver, lungs, spleen, kidney, stomach and intestines. ${ }^{119} 120$ In contrast to intravenous injection, intraperitoneal injection displayed decreased accumulation in the liver, increased distribution in the pancreas and slightly higher whole body accumulation. ${ }^{119}$ Intraperitoneal administration allows larger amount of exosome than other systemic administration routes, but exosomes rapidly diffused in the cavity of peritoneal. ${ }^{121} 122$ In a rat model of perinatal brain injury, dynamic examination of intranasal administrated exosomes prior to ischaemia revealed that exosomes appeared in the frontal brain as early as $30 \mathrm{~min}$ post administration and evenly distributed in the whole brain 3 hours post administration. ${ }^{88}$ Biodistribution analysing intravenous and intranasal delivery of 
exosomes labelled with gold nanoparticles after cerebral ischaemia showed that the amount of intranasal delivered exosomes in the brain was more than twofold higher than intravenous delivery 1-hour post administration. There still were substantial amount of exosomes retaining in the brain after intranasal delivery, while nearly negligible amount after intravenous delivery 24 hours post administration. Importantly, a fair amount of intranasal delivered exosomes also accumulated in the lungs, spleen and kidney. ${ }^{123}$ These results suggested that intranasal delivery was a more efficient and promising non-invasive therapeutic strategy for exosome administration in ischaemic stroke.

Reported local administration is usually the cerebral ventricle injection. ${ }^{89} 106$ In global ischaemia, intracerebroventricular injected MSC exosomes rescued hippocampal synaptic transmission deficits, inhibited cyclo-oxygenase-2 expression and promoted spatial learning and memory. ${ }^{89}$ Additionally, ADSC exosomes delivered via lateral cerebral ventricle for constitutive 3 days before ischaemia ameliorated infarct volume and cell apoptosis 3 days after tMCAO. ${ }^{106}$

\section{EXOSOME LABELLING AND TRACKING Fluorescence microscopy}

Exosomes can be visualised under fluorescence microscopy through directly labelling exosomes with fluorescent dye or indirectly overexpressing fluorescent proteins conjugated to exosomal enriched proteins or miRNAs in parental cells. Lipophilic fluorescent dye such as PKH-26 and Dil-Red can label exosome membrane through inserting aliphatic chains into the lipid bilayer. ${ }^{124}$ A study compared the bio-distribution between liposomes and tumour-derived exosomes labelled with Dil-Red, results revealed that exosomes showed the similar bio-distribution profiles with liposomes in vivo, mostly accumulated in liver and spleen when intravenous administrated and retained in tumour when intratumour injected. ${ }^{125}$ This method is relatively easy to operate. However, the lipophilic fluorescent dye could incorporate with other cellular lipid membranes for long periods and may not reflect the true half-life of exosomes. ${ }^{117} 126$ In order to circumvent this disadvantage, a study labelled exosomes with fluorescent dye using clickchemistry by cross-linking the exosomal membrane proteins with alkyne group. ${ }^{127}$

Another approach is to fuse exosomal membrane protein or exosomal RNA cargos with fluorescent protein reporter gene or peptide, such as enhanced green/red fluorescent protein or Cy3. ${ }^{77} 126$ These can dynamically visualise exosomal spatiotemporal release, uptake and cargo exchange between cells. ${ }^{126}$

\section{Bioluminescent imaging}

Bioluminescent imaging (BLI) is based on light emittingenzyme catalysed reactions to report cellular activity at molecular level. ${ }^{128}{ }^{129}$ Compared with traditional imaging methods, BLI is a powerful imaging technology 
for molecular imaging in living cells and small animals without the need to sacrifice animals. ${ }^{128}$ This enables the investigations of ongoing biological processes in vitro and vivo. One of the most used light emitted enzyme is gaussia luciferase, which can react with its substrate coelenterazine and then emit strong chemiluminescent signal. ${ }^{129} 130$ In order to dynamically detect exosome uptake and mRNA translation in recipient cells, researchers fused exosome mRNA cargos with gaussia luciferase reporter gene. The gaussia luciferase mRNA translation occurred within 1 hour, peaked at 12 hours, declined between 12 and 24 hours and continued up to 72 hours post exosomes delivered to recipient cells. ${ }^{126}$ Systemic administration of exosomes labelled with lactadherin (a membraneassociated protein mainly presented in exosomes) conjugated gaussia luciferase demonstrated similar biodistribution pattern as PKH-26 labelled exosomes, but displayed shorter half-life. ${ }^{120}$ Exosomes displayed rapid distribution and then followed by longer clearance, and the clearance time was much longer in macrophage-depleted mice, suggesting that macrophages might be involved in the clearance of exosomes from circulation system. ${ }^{131}$

\section{MRI}

MRI can provide high-resolution imaging for monitoring stem cells and exosome tracking and distribution in a non-invasive and radiation-free manner. ${ }^{132-135}$ In the first study to track exosome by MRI, exosomes were loaded with $5 \mathrm{~nm}$ superparamagnetic iron oxide nanoparticles through electroporation. Dynamic tracking exosome homing from food pad to local lymph nodes showed that exosomes retained the iron cargo through the whole process for at least 48 hours. ${ }^{136}$ But electroporation induces a temporal disruption of exosomal membrane, which can alter exosomal contents and influence their function. Therefore, another study innovatively incubated ADSCs with ultrasmall superparamagnetic iron oxide nanoparticles then isolated the exosomes. ${ }^{132} 133$ However, the detection limitation of MRI tracking was $3 \mu \mathrm{g}$ and $5 \mu \mathrm{g}$ of exosomes in vitro and in vivo, respectively. ${ }^{133}$

\section{CT}

CT produces images based on the computer processing of X-ray from different angles and is widely used in clinic. A study labelled MSC exosomes with glucose-coated gold nanoparticles and detected the biodistribution of exosome after intranasal administration in the normal and ischaemic brain by CT. In vivo CT imaging showed that exosomes migrated and accumulated in the ischaemic region 3 hours and still within the ischaemic site 24 hours post intranasal administration, while displayed no region specific accumulation and were cleared from the brain after 24 hours in the normal mice. ${ }^{123}$ They further used CT to track the migration and accumulating of intranasal administrated MSC exosomes in stroke, autism, Parkinson's disease and Alzheimer's disease. Results revealed that exosomes specifically targeted and accumulated in the associated pathological brain region up to 96 hours post-delivery, while showed diffused distribution and elimination by 24 hours. These accumulation patterns were related to neuro-inflammation signal. ${ }^{137}$

\section{SPECT and positron emission computed tomography}

SPECT and positron emission CT (PET) are two main clinical nuclear imaging tools, and acquire images by detecting gamma rays generated directly from gammaemitting radioisotopes and indirectly from positronemitting radionuclide, respectively. ${ }^{138}$ Different from MRI and CT emphasising on anatomy imaging, nuclear imaging is a molecular and functional imaging that can visualise and measure bimolecular metabolism and cellular activity throughout the whole-body. ${ }^{139} 140$ Compared with SPECT, PET imaging has higher resolution and sensitivity. ${ }^{140}$ The major advantages of nuclear imaging are the unlimited tissue penetration and quantitative analysis with the shortcoming of low tissue anatomical information. ${ }^{139}$ Therefore, they are usually combined with CT or MRI. Dynamic visualising intravenously injected ${ }^{99} \mathrm{mTc}$-HMPAO labelled exosomes in normal mice by SPECT/CT showed that the uptake of exosomes were mainly in liver and spleen 30 min postinjection, and in salivary glands 3 hours post-injection, but very few in the brain at any time point. ${ }^{141} \mathrm{Up}$ to date, the SPECT/CT and PET imaging of exosomes labelled with radiotracers are mainly focused on tumour and no reports in cerebral ischaemia. ${ }^{142-144}$ For example, tumour cell derived exosomes were labelled with iodine-131for SPECT/CT imaging and ex vivo gamma counter could quantitatively evaluate the amount of exosomes delivered to each organ. ${ }^{144}$ Another study labelled breast cancer cell derived exosomes with copper-64 for PET imaging and polyethylene glycol for enhanced in vivo properties. The accuracy of PET imaging of intravenous delivered exosome tissue biodistribution was confirmed by ex vivo gamma counting. ${ }^{143}$ These suggested than SPECT and PET are potentially imaging methods for exosome tracking in vivo and can pave the way for the development of future mechanism researches, therapeutic and diagnostic applications.

\section{EXOSOME TARGETING}

Exosome targeting has emerged as an innovative and important engineering strategy for enhancing and optimising therapeutic effects by targeting specific tissue and cell type. The basic principle of exosome targeting is to fuse specific tissue homing or cell targeting peptide to exosome membrane proteins, such as Lamp2b. A study fused neuron-specific RVG peptide (specifically binds to the acetylcholine receptor) to Lamp2b and isolated exosomes form dendritic cells overexpressing fused Lamp2b. Then these modified exosomes were loaded with exogenous glyceraldehyde-3-phosphate dehydrogenase (GAPDH) short interfering RNA (siRNA) by electroporation. Intravenously injected GAPDH siRNA loaded plus RVG-fused exosomes specifically knockdown 
GAPDH expression in striatum, midbrain and cortex, but not in the liver, muscle, heart and other organs. Further examination of the colocalisation of siRNA and brain cell specific marker revealed that RVG-conjugated exosomes targeted primarily to neurons, microglia, oligodendrocytes and their precursors. ${ }^{145}$ Therefore, intravenous administration of RVG modified MSC exosomes 1-day post cerebral ischaemia exerted greater therapeutic effects on promoting neurogenesis and reducing brain injury. ${ }^{98}$ To specifically target brain endothelial cells, MSC exosomes were conjugated with the cycle (Arg-GlyAsp-D-Tyr-Lys) peptide (c(RGDyK)) using clickchemistry, which had high affinity to integrin $\alpha_{v} \beta_{3}$ in reactive cerebral endothelial cells after ischaemia. Analysing the fluorescence intensity of intravenous injected exosomes in different organs showed that compared with unmodified exosomes, $\mathrm{c}(\mathrm{RGDyK})$ modification increased the amount of exosomes in the brain, liver and lung, and the amount in the brain was higher than that in the liver and lung, suggesting that $\mathrm{c}(\mathrm{RGDyK})$ conjugation enhanced exosomal tropism to ischaemic brain region. Further administration of curcumin loaded c(RGDyK) exosomes to ischaemic mice effectively inhibited inflammation and cellular apoptosis in ischaemic region compared with control groups. ${ }^{146}$ In mice ischaemia, MSC exosomes were modified with $\mathrm{c}$ (RGDyK) and loaded with miR-210 (RGD-exo:miR-210). Intravenously injected RGD modified exosomes mainly accumulated in the ipsilateral brain and liver, RGD-exo:miR-210 more potently upregulated VEGF, integrin $\beta 3$ expression and promoted angiogenesis compared with RGD modified exosomes. ${ }^{147}$ EGFR could also serve as a specific target for tumour and ischaemic region with angiogenesis. GE11 peptide (amino-acid sequence YHWYGYTPQNVI) specifically binds to EGFR, but less mitogenic than EGF. The uptake of EGF and GE11 peptide modified exosomes showed an EGFR concentration-dependent manner, but EGF modified exosomes stimulated cellular EGFR signalling. ${ }^{148}$

Recently, new methods have been developed to further enhance exosomes targeting. In a study, researchers engineered exosome membrane to create a membraneanchoring platform termed 'cloaking', which could provide anchoring sites to embed any biotinylated tissuespecific antibodies or homing peptides onto exosomes. Examination the uptake of the engineered exosomes with cardiac fibroblasts target bio-DR, muscle targeting peptide MTP and ischaemic targeting peptide sequence CSTSMLKAC showed specific target cell and tissue accumulation and homing than control group. ${ }^{149}$ Another way to load functional proteins into exosomes was to fuse the target protein with a WW tag, which could be recognise by L-domain-containing protein Ndfip1 and induce ubiquitination and protein loading into exosomes. ${ }^{150}$ Collectively, these researches enabled the application of modulating exosome tropism and targeting to improve exosome treatment efficiency.

\section{EXOSOME CLINICAL TRIALS IN STROKE}

Many preliminary works have proven that exosomes were sufficient to promote neurovascular remodelling and functional recovery in ischaemic stroke. However, the clinical investigations of exosome-based therapy in stroke have just begun to explore, and well-designed clinical trials are warranted to promote exosome translation to clinic. Up to now, only one registered clinical trial NCT03384433 was reported in exosome treatment of stroke. Researchers are aiming to evaluate the administration of allogenic MSC exosomes enriched with miR-124 on improvement of disability in patients with acute ischaemic stroke. This clinical trial is still under recruiting, and patients will receive $200 \mathrm{mg}$ exosomes from MSC transfected by miR-124 via stereotaxic administration 1 month after ischaemia onset. Measurement of modified ranking scale and adverse treatment related events will be conducted 12 months post exosome administration. Due to the therapeutic mechanisms of exosomes are largely mediated by miRNAs, we also reviewed miRNA clinical trials in stroke. Noteworthily, most of miRNA clinical trials are focused on the prediction, diagnosis and prognosis roles of miRNAs in stroke research. For example, in order to identify serum miRNAs for the stroke risk prediction, researchers finally examined and analysed the serum miRNAs from 1523 controls aged 40-69 years and 173 cerebrovascular disorder cases aged over 69 years. Using three step strategy, they identified 10 miRNAs correlated with a predicted risk of stroke, 7 of which were significantly associated with cerebrovascular disorder. Finally, they constructed a 3-miRNA (miR1268b, miR-4433b-3p and miR-6803-5p) combination model for risk predicting before stroke onset. ${ }^{151}$ Clinical trial NCT04175691 and NCT04230785 will analyse the expression pattern of circular RNA, miRNAs and long non-coding RNA using next-generation sequencing in patients to verify related biomarkers for detection and prognosis of acute ischaemic stroke, and for progression and prognosis of acute ischaemic stroke with endovascular treatment, respectively. Importantly, one on recruiting study NCT03577093 tries to investigate the molecular mechanisms of microRNA-494 mediating cell cycle regulation after cerebral ischaemia. They designed to recruit patients who had a stroke within 6 hours onset aged 18-80 years and examine peripheral blood DNA samples.

\section{CONCLUSIONS AND FUTURE PERSPECTIVES}

Exosomes have gained increasing interest in cerebral ischaemia and have been considered to be potential therapeutic strategy. Numerous evidence demonstrates that exosomes derived from either stem cells or other cells exert protective and restorative effects in stroke. Furthermore, engineered exosomes exhibit greater therapeutic benefits. Therefore, exosomes could be tailed specifically with restorative genes, proteins, drugs and molecules to obtain better efficacy. However, the dose, frequency and routes of exosome delivery still do not have concise 
agreement and need further investigation to accelerate exosome-based therapy from bench to bedside. The development of exosome tracking and targeting will advance our understanding of exogenous exosome biodistribution, pharmacokinetics and further promoting therapeutic effects and clinical translation of exosomebased therapy in stroke.

Contributors $\mathrm{YL}$ is responsible for drafting the manuscript, $\mathrm{YT}$ and G-YY are involved in outlining and revising the work for critical intellectual content.

Funding This study was funded by 2019YFA0112000, NSFC 81801170 , ZH2018QNA16, ZH2018ZDA04.

Competing interests None declared.

Patient consent for publication Not required.

Provenance and peer review Commissioned; externally peer reviewed.

Open access This is an open access article distributed in accordance with the Creative Commons Attribution Non Commercial (CC BY-NC 4.0) license, which permits others to distribute, remix, adapt, build upon this work non-commercially, and license their derivative works on different terms, provided the original work is properly cited, appropriate credit is given, any changes made indicated, and the use is non-commercial. See: http://creativecommons.org/licenses/by-nc/4.0/.

\section{ORCID iD}

Guo-Yuan Yang http://orcid.org/0000-0003-3105-9307

\section{REFERENCES}

1 Zhou $\mathrm{M}$, Wang $\mathrm{H}$, Zeng $\mathrm{X}$, et al. Mortality, morbidity, and risk factors in China and its provinces, 1990-2017: a systematic analysis for the global burden of disease study 2017. Lancet 2019;394:1145-58

2 Benjamin EJ, Blaha MJ, Chiuve SE, et al. Heart disease and stroke Statistics-2017 update: a report from the American heart association. Circulation 2017;135:e146-603.

3 Hankey GJ. Stroke. Lancet 2017;389:641-54

4 Zhang ZG, Buller B, Chopp M. Exosomes - beyond stem cells for restorative therapy in stroke and neurological injury. Nat Rev Neurol 2019;15:193-203.

5 Venkat P, Chen J, Chopp M. Exosome-Mediated amplification of endogenous brain repair mechanisms and brain and systemic organ interaction in modulating neurological outcome after stroke. $J$ Cereb Blood Flow Metab 2018;38:2165-78.

6 Chen J, Chopp M. Exosome therapy for stroke. Stroke 2018;49:1083-90.

7 Kalluri R, LeBleu VS. The biology, function, and biomedical applications of exosomes. Science 2020;367. doi:10.1126/science. aau6977. [Epub ahead of print: 07 Feb 2020].

8 Zhang ZG, Chopp M. Exosomes in stroke pathogenesis and therapy. J Clin Invest 2016;126:1190-7.

9 Zhang J, Li S, Li L, et al. Exosome and exosomal microRNA: trafficking, sorting, and function. Genomics Proteomics Bioinformatics 2015:13:17-24.

10 Eulalio A, Huntzinger E, Izaurralde E. Getting to the root of miRNAmediated gene silencing. Cell 2008;132:9-14.

11 Li Y, Yang G-Y. Pathophysiology of Ischemic Stroke. In: Lapchak PA, Yang G-Y, eds. Translational research in stroke. Singapore: Springer Singapore, 2017: 51-75.

12 Khoshnam SE, Winlow W, Farzaneh M, et al. Pathogenic mechanisms following ischemic stroke. Neurol Sci 2017;38:1167-86.

13 Zhao L-R, Willing A. Enhancing endogenous capacity to repair a stroke-damaged brain: an evolving field for stroke research. Prog Neurobiol 2018:163-164:5-26.

14 Ruan L, Wang B, ZhuGe Q, et al. Coupling of neurogenesis and angiogenesis after ischemic stroke. Brain Res 2015;1623:166-73.

15 Shen F, Jiang L, Han F, et al. Increased inflammatory response in old mice is associated with more severe neuronal injury at the acute stage of ischemic stroke. Aging Dis 2019;10:12-22.

16 Jayaraj RL, Azimullah S, Beiram R, et al. Neuroinflammation: Friend and foe for ischemic stroke. J Neuroinflammation 2019;16:142.

17 Rust R, Grönnert L, Schwab ME. Inflammation after stroke: a local rather than systemic response? Trends Neurosci 2018;41:877-9.
18 Nakamura K, Shichita T. Cellular and molecular mechanisms of sterile inflammation in ischaemic stroke. $J$ Biochem 2019;165:459-64.

19 Tsuyama J, Nakamura A, Ooboshi H, et al. Pivotal role of innate myeloid cells in cerebral post-ischemic sterile inflammation. Semin Immunopathol 2018;40:523-38.

20 Ma Y, Liu Y, Zhang Z, et al. Significance of complement system in ischemic stroke: a comprehensive review. Aging Dis 2019;10:429-62.

21 Ao L-Y, Yan Y-Y, Zhou L, et al. Immune cells after ischemic stroke onset: roles, migration, and target intervention. $J$ Mol Neurosci 2018:66:342-55.

22 Rayasam A, Hsu M, Kijak JA, et al. Immune responses in stroke: how the immune system contributes to damage and healing after stroke and how this knowledge could be translated to better cures? Immunology 2018;154:363-76.

23 Haley MJ, Lawrence CB. The blood-brain barrier after stroke: structural studies and the role of transcytotic vesicles. J Cereb Blood Flow Metab 2017;37:456-70.

24 Keaney J, Campbell M. The dynamic blood-brain barrier. Febs J 2015;282:4067-79.

25 Krueger M, Mages B, Hobusch C, et al. Endothelial edema precedes blood-brain barrier breakdown in early time points after experimental focal cerebral ischemia. Acta Neuropathol Commun 2019;7:17.

26 Jiang X, Andjelkovic AV, Zhu L, et al. Blood-Brain barrier dysfunction and recovery after ischemic stroke. Prog Neurobiol 2018;163-164:144-71.

27 Cheng Z, Wang L, Qu M, et al. Mesenchymal stem cells attenuate blood-brain barrier leakage after cerebral ischemia in mice. $J$ Neuroinflammation 2018:15:135.

28 Abdullahi W, Tripathi D, Ronaldson PT. Blood-Brain barrier dysfunction in ischemic stroke: targeting tight junctions and transporters for vascular protection. Am J Physiol Cell Physiol 2018;315:C343-56.

29 Deng X-X, Li S-S, Sun F-Y. Necrostatin-1 prevents necroptosis in brains after ischemic stroke via inhibition of RIPK1-Mediated RIPK3/MLKL signaling. Aging Dis 2019;10:807-17.

30 Rami A, Kögel D. Apoptosis meets autophagy-like cell death in the ischemic penumbra: two sides of the same coin? Autophagy 2008;4:422-6.

31 Wang P, Shao B-Z, Deng Z, et al. Autophagy in ischemic stroke. Prog Neurobiol 2018;163-164:98-117.

32 Datta A, Sarmah D, Mounica L, et al. Cell death pathways in ischemic stroke and targeted pharmacotherapy. Trans/ Stroke Res 2020. doi:10.1007/s12975-020-00806-z. [Epub ahead of print: 26 Mar 2020].

33 Loh KY, Wang Z, Liao P. Oncotic cell death in stroke. Rev Physiol Biochem Pharmacol 2019;176:37-64.

34 Sekerdag E, Solaroglu I, Gursoy-Ozdemir Y. Cell death mechanisms in stroke and novel molecular and cellular treatment options. Curr Neuropharmacol 2018;16:1396-415.

35 Li P, Stetler RA, Leak RK, et al. Oxidative stress and DNA damage after cerebral ischemia: potential therapeutic targets to repair the genome and improve stroke recovery. Neuropharmacology 2018;134:208-17.

36 Fricker M, Tolkovsky AM, Borutaite V, et al. Neuronal cell death. Physiol Rev 2018;98:813-80.

37 Arumugam TV, Baik S-H, Balaganapathy P, et al. Notch signaling and neuronal death in stroke. Prog Neurobiol 2018;165167:103-16

38 Chamorro Ángel, Dirnagl U, Urra X, et al. Neuroprotection in acute stroke: targeting excitotoxicity, oxidative and nitrosative stress, and inflammation. Lancet Neurol 2016;15:869-81.

39 Kanazawa M, Takahashi T, Ishikawa M, et al. Angiogenesis in the ischemic core: a potential treatment target? J Cereb Blood Flow Metab 2019;39:753-69.

40 Rosell A, Ortega-Aznar A, Alvarez-Sabín J, et al. Increased brain expression of matrix metalloproteinase-9 after ischemic and hemorrhagic human stroke. Stroke 2006;37:1399-406.

41 Clark AW, Krekoski CA, Bou SS, et al. Increased gelatinase A (MMP-2) and gelatinase B (MMP-9) activities in human brain after focal ischemia. Neurosci Lett 1997;238:53-6.

42 Mao L, Huang M, Chen S-C, et al. Endogenous endothelial progenitor cells participate in neovascularization via CXCR4/ SDF-1 axis and improve outcome after stroke. CNS Neurosci Ther 2014;20:460-8.

43 Zhang ZG, Zhang L, Jiang Q, et al. Bone marrowderived endothelial progenitor cells participate in cerebral neovascularization after focal cerebral ischemia in the adult mouse. Circ Res 2002;90:284-8. 
44 Marques BL, Carvalho GA, Freitas EMM, et al. The role of neurogenesis in neurorepair after ischemic stroke. Semin Cell Dev Biol 2019;95:98-110.

45 Gross CG. Neurogenesis in the adult brain: death of a dogma. Nat Rev Neurosci 2000;1:67-73.

46 Li W, Wang Y. Angiogenesis and Neurogenesis After Ischemic Stroke. In: Lapchak PA, Yang G-Y, eds. Translational research in stroke. Singapore: Springer Singapore, 2017: 297-317.

47 Thored P, Wood J, Arvidsson A, et al. Long-Term neuroblast migration along blood vessels in an area with transient angiogenesis and increased vascularization after stroke. Stroke 2007;38:3032-9.

48 Duan C-L, Liu C-W, Shen S-W, et al. Striatal astrocytes transdifferentiate into functional mature neurons following ischemic brain injury. Glia 2015;63:1660-70.

49 Barker RA, Götz M, Parmar M. New approaches for brain repairfrom rescue to reprogramming. Nature 2018;557:329-34.

50 Niu W, Zang T, Zou Y, et al. In vivo reprogramming of astrocytes to neuroblasts in the adult brain. Nat Cell Biol 2013;15:1164-75.

51 Tanaka K, Nogawa S, Suzuki S, et al. Upregulation of oligodendrocyte progenitor cells associated with restoration of mature oligodendrocytes and myelination in peri-infarct area in the rat brain. Brain Res 2003;989:172-9.

52 Jiang L, Shen F, Degos V, et al. Oligogenesis and oligodendrocyte progenitor maturation vary in different brain regions and partially correlate with local angiogenesis after ischemic stroke. Trans/ Stroke Res 2011;2:366-75.

53 van Niel G, D'Angelo G, Raposo G. Shedding light on the cell biology of extracellular vesicles. Nat Rev Mol Cell Biol 2018;19:213-28.

54 Johnstone RM, Adam M, Hammond JR, et al. Vesicle formation during reticulocyte maturation. Association of plasma membrane activities with released vesicles (exosomes). J Biol Chem 1987;262:9412-20.

55 Pan BT, Teng K, Wu C, et al. Electron microscopic evidence for externalization of the transferrin receptor in vesicular form in sheep reticulocytes. J Cell Biol 1985;101:942-8.

56 Pan BT, Johnstone RM. Fate of the transferrin receptor during maturation of sheep reticulocytes in vitro: selective externalization of the receptor. Cell 1983;33:967-78.

57 Kowal J, Tkach M, Théry C. Biogenesis and secretion of exosomes. Curr Opin Cell Biol 2014;29:116-25.

58 Zitvogel L, Regnault A, Lozier A, et al. Eradication of established murine tumors using a novel cell-free vaccine: dendritic cell-derived exosomes. Nat Med 1998;4:594-600.

59 Raposo G, Nijman HW, Stoorvogel W, et al. B lymphocytes secrete antigen-presenting vesicles. J Exp Med 1996;183:1161-72.

60 Valadi H, Ekström K, Bossios A, et al. Exosome-Mediated transfer of mRNAs and microRNAs is a novel mechanism of genetic exchange between cells. Nat Cell Biol 2007;9:654-9.

61 Colombo M, Raposo G, Théry C. Biogenesis, secretion, and intercellular interactions of exosomes and other extracellula vesicles. Annu Rev Cell Dev Biol 2014;30:255-89.

62 Kordelas L, Rebmann V, Ludwig A-K, et al. Msc-Derived exosomes: a novel tool to treat therapy-refractory graft-versus-host disease. Leukemia 2014;28:970-3.

63 Hong S-B, Yang H, Manaenko A, et al. Potential of exosomes for the treatment of stroke. Cell Transplant 2019;28:662-70.

64 Das CK, Jena BC, Banerjee I, et al. Exosome as a novel shuttle for delivery of therapeutics across biological barriers. Mol Pharm 2019;16:24-40.

65 Otero-Ortega L, Laso-García F, Gómez-de Frutos M, et al. Role of exosomes as a treatment and potential biomarker for stroke. Trans Stroke Res 2019;10:241-9.

66 Liu W, Bai X, Zhang A, et al. Role of exosomes in central nervous system diseases. Front Mol Neurosci 2019;12:240.

67 Datta A, Chen CP, Sze SK. Discovery of prognostic biomarker candidates of lacunar infarction by quantitative proteomics of microvesicles enriched plasma. PLoS One 2014;9:e94663.

68 Kerr N, García-Contreras M, Abbassi S, et al. Inflammasome proteins in serum and serum-derived extracellular vesicles as biomarkers of stroke. Front Mol Neurosci 2018;11:309.

69 Kanhai DA, de Kleijn DPV, Kappelle LJ, et al. Extracellular vesicle protein levels are related to brain atrophy and cerebral white matter lesions in patients with manifest vascular disease: the SMART-MR study. BMJ Open 2014;4:e003824.

70 Chen F, Du Y, Esposito E, et al. Effects of focal cerebral ischemia on exosomal versus serum miR126. Trans/ Stroke Res 2015;6:478-84.

71 Ji Q, Ji Y, Peng J, et al. Increased brain-specific miR-9 and miR-124 in the serum exosomes of acute ischemic stroke patients. PLoS One 2016;11:e0163645.
72 Zhou J, Chen L, Chen B, et al. Increased serum exosomal miR-134 expression in the acute ischemic stroke patients. BMC Neurol 2018;18:198.

73 Chen Y, Song Y, Huang J, et al. Increased circulating exosomal miRNA-223 is associated with acute ischemic stroke. Front Neurol 2017;8:57.

74 Li D-B, Liu J-L, Wang W, et al. Plasma exosomal miR-422a and miR-125b-2-3p serve as biomarkers for ischemic stroke. Curr Neurovasc Res 2017;14:330-7.

75 Wang W, Li D-B, Li R-Y, et al. Diagnosis of hyperacute and acute ischaemic stroke: the potential utility of exosomal MicroRNA-21-5p and MicroRNA-30a-5p. Cerebrovasc Dis 2018;45:204-12.

76 Wang J, Liu H, Chen S, et al. Moderate exercise has beneficial effects on mouse ischemic stroke by enhancing the functions of circulating endothelial progenitor cell-derived exosomes. Exp Neurol 2020;330:113325

77 Hou Z, Qin X, Hu Y, et al. Longterm Exercise-Derived exosomal miR-342-5p: a novel Exerkine for cardioprotection. Circ Res 2019;124:1386-400.

78 Hira K, Ueno Y, Tanaka R, et al. Astrocyte-Derived Exosomes Treated With a Semaphorin 3A Inhibitor Enhance Stroke Recovery via Prostaglandin D Synthase. Stroke 2018;49:2483-94.

79 Deng L, Peng Q, Wang H, et al. Intrathecal injection of allogenic bone marrow-derived mesenchymal stromal cells in treatment of patients with severe ischemic stroke: study protocol for a randomized controlled Observer-Blinded trial. Trans/ Stroke Res 2019;10:170-7

80 Xin H, Li Y, Cui Y, et al. Systemic administration of exosomes released from mesenchymal stromal cells promote functional recovery and neurovascular plasticity after stroke in rats. $J$ Cereb Blood Flow Metab 2013;33:1711-5.

$81 \mathrm{Hu} \mathrm{B}$, Chen S, Zou M, et al. Effect of extracellular vesicles on neura functional recovery and immunologic suppression after rat cerebral apoplexy. Cell Physiol Biochem 2016;40:155-62.

82 Doeppner TR, Herz J, Görgens A, et al. Extracellular vesicles improve post-stroke neuroregeneration and prevent postischemic immunosuppression. Stem Cells Transl Med 2015;4:1131-43.

83 Lee JY, Kim E, Choi S-M, et al. Microvesicles from brain-extracttreated mesenchymal stem cells improve neurological functions in a rat model of ischemic stroke. Sci Rep 2016;6:33038.

84 Nalamolu KR, Venkatesh I, Mohandass A et al. Exosomes secreted by the cocultures of normal and oxygen-glucose-deprived stem cells improve post-stroke outcome. Neuromolecular Med 2019;21:529-39.

85 Go V, Bowley BGE, Pessina MA, et al. Extracellular vesicles from mesenchymal stem cells reduce microglial-mediated neuroinflammation after cortical injury in aged rhesus monkeys. Geroscience 2020;42:1-17.

86 Medalla M, Chang W, Calderazzo SM, et al. Treatment with mesenchymal-derived extracellular vesicles reduces injury-related pathology in pyramidal neurons of monkey perilesional ventral premotor cortex. J Neurosci 2020;40:3385-407.

87 Thomi G, Surbek D, Haesler V, et al. Exosomes derived from umbilical cord mesenchymal stem cells reduce microglia-mediated neuroinflammation in perinatal brain injury. Stem Cell Res Ther 2019;10:105.

88 Thomi G, Joerger-Messerli M, Haesler V, et al. Intranasally administered exosomes from umbilical cord stem cells have preventive neuroprotective effects and contribute to functional recovery after perinatal brain injury. Cells 2019;8. doi:10.3390/ cells8080855. [Epub ahead of print: 08 Aug 2019].

89 Deng $\mathrm{M}$, Xiao $\mathrm{H}$, Zhang $\mathrm{H}$, et al. Mesenchymal stem cell-derived extracellular vesicles ameliorates hippocampal synaptic impairment after transient global ischemia. Front Cell Neurosci 2017;11:205

90 Otero-Ortega L, Laso-García F, Gómez-de Frutos MDC, et al. White matter repair after extracellular vesicles administration in an experimental animal model of subcortical stroke. Sci Rep 2017;7:44433.

91 Zhang Y, Chopp M, Liu XS, et al. Exosomes derived from mesenchymal stromal cells promote axonal growth of cortical neurons. Mol Neurobiol 2017;54:2659-73.

92 Xiao Y, Geng F, Wang G, et al. Bone marrow-derived mesenchymal stem cells-derived exosomes prevent oligodendrocyte apoptosis through exosomal miR-134 by targeting caspase-8. Journal of Cellular Biochemistry 2019;120.

93 Xin H, Li Y, Liu Z, et al. Mir-133B promotes neural plasticity and functional recovery after treatment of stroke with multipotent mesenchymal stromal cells in rats via transfer of exosome-enriched extracellular particles. Stem Cells 2013;31:2737-46.

94 Joerger-Messerli MS, Oppliger B, Spinelli M, et al. Extracellular vesicles derived from Wharton's jelly mesenchymal stem cells 
prevent and resolve programmed cell death mediated by perinatal hypoxia-ischemia in neuronal cells. Cell Transplant 2018;27:168-80.

95 Tran PHL, Xiang D, Tran TTD, et al. Exosomes and Nanoengineering: a match made for precision therapeutics. Adv Mater 2020;32:e1904040.

96 Luan X, Sansanaphongpricha K, Myers I, et al. Engineering exosomes as refined biological nanoplatforms for drug delivery. Acta Pharmacol Sin 2017;38:754-63.

97 Xin H, Katakowski M, Wang F, et al. Microrna cluster miR-1792 cluster in exosomes enhance neuroplasticity and functional recovery after stroke in rats. Stroke 2017;48:747-53.

98 Yang J, Zhang X, Chen X, et al. Exosome mediated delivery of miR124 promotes neurogenesis after ischemia. Mol Ther Nucleic Acids 2017;7:278-87.

99 Webb RL, Kaiser EE, Scoville SL, et al. Human neural stem cell extracellular vesicles improve tissue and functional recovery in the murine thromboembolic stroke model. Trans/ Stroke Res 2018;9:530-9.

100 Sun X, Jung J-H, Arvola O, et al. Stem Cell-Derived Exosomes Protect Astrocyte Cultures From in vitro Ischemia and Decrease Injury as Post-stroke Intravenous Therapy. Front Cell Neurosci 2019;13:394.

101 Webb RL, Kaiser EE, Jurgielewicz BJ, et al. Human neural stem cell extracellular vesicles improve recovery in a porcine model of ischemic stroke. Stroke 2018:49:1248-56.

102 Hong P, Yang H, Wu Y, et al. The functions and clinical application potential of exosomes derived from adipose mesenchymal stem cells: a comprehensive review. Stem Cell Res Ther 2019;10:242.

103 Yang Y, Cai Y, Zhang Y, et al. Exosomes secreted by adiposederived stem cells contribute to angiogenesis of brain microvascular endothelial cells following oxygen-glucose deprivation in vitro through MicroRNA-181b/TRPM7 axis. J Mol Neurosci 2018;65:74-83

104 Geng W, Tang H, Luo S, et al. Exosomes from miRNA-126-modified ADSCs promotes functional recovery after stroke in rats by improving neurogenesis and suppressing microglia activation. Am J Transl Res 2019;11:780-92.

105 Jiang $M$, Wang $\mathrm{H}$, Jin $\mathrm{M}$, et al. Exosomes from MiR-30d-5p-ADSCs reverse acute ischemic stroke-induced, autophagy-mediated brain injury by promoting M2 Microglial/Macrophage polarization. Cell Physiol Biochem 2018;47:864-78.

106 Huang X, Ding J, Li Y, et al. Exosomes derived from PEDF modified adipose-derived mesenchymal stem cells ameliorate cerebral ischemia-reperfusion injury by regulation of autophagy and apoptosis. Exp Cell Res 2018;371:269-77.

107 Pei X, Li Y, Zhu L, et al. Astrocyte-Derived exosomes suppress autophagy and ameliorate neuronal damage in experimental ischemic stroke. Exp Cell Res 2019;382:111474.

108 Xu L, Cao H, Xie Y, et al. Exosome-shuttled miR-92b-3p from ischemic preconditioned astrocytes protects neurons against oxygen and glucose deprivation. Brain Res 2019;1717:66-73.

109 Pan Q, He C, Liu H, et al. Microvascular endothelial cells-derived microvesicles imply in ischemic stroke by modulating astrocyte and blood brain barrier function and cerebral blood flow. Mol Brain 2016;9:63.

110 Wang X, Wang J, Shi X, et al. Proteomic analyses identify a potential mechanism by which extracellular vesicles aggravate ischemic stroke. Life Sci 2019;231:116527.

$111 \mathrm{Li} \mathrm{Y,} \mathrm{Ren} \mathrm{C,} \mathrm{Li} \mathrm{H,} \mathrm{et} \mathrm{al.} \mathrm{Role} \mathrm{of} \mathrm{exosomes} \mathrm{induced} \mathrm{by} \mathrm{remote}$ ischemic preconditioning in neuroprotection against cerebral ischemia. Neuroreport 2019;30:834-41.

112 Xiao B, Chai Y, Lv S, et al. Endothelial cell-derived exosomes protect SH-SY5Y nerve cells against ischemia/reperfusion injury. Int J Mol Med 2017;40:1201-9.

113 Njock M-S, Cheng HS, Dang LT, et al. Endothelial cells suppress monocyte activation through secretion of extracellular vesicles containing antiinflammatory microRNAs. Blood 2015;125:3202-12.

114 Venkat $\mathrm{P}$, Cui C, Chopp M, et al. Mir-126 mediates brain endothelial cell exosome treatment-induced neurorestorative effects after stroke in type 2 diabetes mellitus mice. Stroke 2019;50:2865-74.

115 Tian Y, Zhu P, Liu S, et al. IL-4-polarized BV2 microglia cells promote angiogenesis by secreting exosomes. Adv Clin Exp Med 2019;28:421-30.

116 Song Y, Li Z, He T, et al. M2 microglia-derived exosomes protect the mouse brain from ischemia-reperfusion injury via exosomal miR124. Theranostics 2019;9:2910-23.

117 Lai CP, Mardini O, Ericsson M, et al. Dynamic biodistribution of extracellular vesicles in vivo using a multimodal imaging reporter. ACS Nano 2014;8:483-94.

118 Morishita M, Takahashi Y, Nishikawa M, et al. Quantitative analysis of tissue distribution of the B16BL6-derived exosomes using a streptavidin-lactadherin fusion protein and iodine-125-labeled biotin derivative after intravenous injection in mice. J Pharm Sci 2015;104:705-13

119 Wiklander OPB, Nordin JZ, O'Loughlin A, et al. Extracellular vesicle in vivo biodistribution is determined by cell source, route of administration and targeting. J Extracell Vesicles 2015;4:26316.

120 Takahashi Y, Nishikawa M, Shinotsuka $\mathrm{H}$, et al. Visualization and in vivo tracking of the exosomes of murine melanoma B16-BL6 cells in mice after intravenous injection. J Biotechnol 2013;165:77-84.

121 Rezaie J, Ajezi S, Avci Çığır Biray, et al. Exosomes and their application in biomedical field: difficulties and advantages. $\mathrm{Mol}$ Neurobiol 2018:55:3372-93

122 Pinheiro A, Silva AM, Teixeira JH, et al. Extracellular vesicles: intelligent delivery strategies for therapeutic applications. J Control Release 2018;289:56-69.

123 Betzer O, Perets N, Angel A, et al. In vivo neuroimaging of exosomes using gold nanoparticles. ACS Nano 2017:11:10883-93.

124 Ter-Ovanesyan D, Kowal EJK, Regev A, et al. Imaging of isolated extracellular vesicles using fluorescence microscopy. Methods $\mathrm{Mol}$ Biol 2017;1660:233-41.

125 Smyth T, Kullberg M, Malik N, et al. Biodistribution and delivery efficiency of unmodified tumor-derived exosomes. J Control Release 2015;199:145-55

126 Lai CP, Kim EY, Badr CE, et al. Visualization and tracking of tumour extracellular vesicle delivery and RNA translation using multiplexed reporters. Nat Commun 2015;6:7029.

127 Smyth T, Petrova K, Payton NM, et al. Surface functionalization of exosomes using click chemistry. Bioconjug Chem 2014;25:1777-84.

128 Tung JK, Berglund K, Gutekunst C-A, et al. Bioluminescence imaging in live cells and animals. Neurophotonics 2016;3:1.

129 Sato A, Klaunberg B, Tolwani R. In vivo bioluminescence imaging. Comp Med 2004;54:631-4.

130 Zhang K, Li Z. Molecular imaging of therapeutic effect of mesenchymal stem cell-derived exosomes for hindlimb ischemia treatment. Methods Mol Biol. 2019.

131 Imai T, Takahashi Y, Nishikawa M, et al. Macrophage-Dependent clearance of systemically administered B16BL6-derived exosomes from the blood circulation in mice. J Extracell Vesicles 2015;4:26238

132 Busato A, Bonafede R, Bontempi P, et al. Labeling and magnetic resonance imaging of exosomes isolated from adipose stem cells. Curr Protoc Cell Biol 2017;75:3.44.1-3.44.15.

133 Busato A, Bonafede R, Bontempi P, et al. Magnetic resonance imaging of ultrasmall superparamagnetic iron oxide-labeled exosomes from stem cells: a new method to obtain labeled exosomes. Int J Nanomedicine 2016;11:2481.

134 Nguyen PK, Riegler J, Wu JC. Stem cell imaging: from bench to bedside. Cell Stem Cell 2014;14:431-44.

135 Neri M, Maderna C, Cavazzin C, et al. Efficient in vitro labeling of human neural precursor cells with superparamagnetic iron oxide particles: relevance for in vivo cell tracking. Stem Cells 2008;26:505-16.

$136 \mathrm{Hu}$ L, Wickline SA, Hood JL. Magnetic resonance imaging of melanoma exosomes in lymph nodes. Magn Reson Med 2015;74:266-71.

137 Perets N, Betzer O, Shapira R, et al. Golden exosomes selectively target brain pathologies in neurodegenerative and neurodevelopmental disorders. Nano Lett 2019;19:3422-31.

138 Chuo ST-Y, Chien JC-Y, Lai CP-K. Imaging extracellular vesicles: current and emerging methods. J Biomed Sci 2018;25:91:91.

139 Van Der Naalt J. Resting functional imaging tools (MRS, SPECT, PET and PCT). Handb Clin Neurol 2015;127:295-308.

140 Zhu L, Ploessl K, Kung HF. PET/SPECT imaging agents for neurodegenerative diseases. Chem Soc Rev 2014:43:6683-91.

141 Hwang DW, Choi H, Jang SC, et al. Noninvasive imaging of radiolabeled exosome-mimetic nanovesicle using (99m)Tc-HMPAO. Sci Rep 2015;5:15636.

142 Gangadaran P, Hong CM, Ahn B-C. Current perspectives on in vivo noninvasive tracking of extracellular vesicles with molecular imaging. Biomed Res Int 2017;2017:1-11.

143 Shi S, Li T, Wen X, et al. Copper-64 labeled PEGylated exosomes for in vivo positron emission tomography and enhanced tumor retention. Bioconjug Chem 2019;30:2675-83.

144 Rashid MH, Borin TF, Ara R, et al. Differential in vivo biodistribution of ${ }^{131}$ I-labeled exosomes from diverse cellular origins and its implication for theranostic application. Nanomedicine 2019;21:102072.

145 Alvarez-Erviti L, Seow Y, Yin H, et al. Delivery of siRNA to the mouse brain by systemic injection of targeted exosomes. Nat Biotechnol 2011;29:341-5. 
146 Tian T, Zhang H-X, He C-P, et al. Surface functionalized exosomes as targeted drug delivery vehicles for cerebral ischemia therapy. Biomaterials 2018;150:137-49.

147 Zhang H, Wu J, Wu J, et al. Exosome-Mediated targeted delivery of miR-210 for angiogenic therapy after cerebral ischemia in mice. $J$ Nanobiotechnology 2019;17:29.

148 Ohno S-ichiro, Takanashi M, Sudo K, et al. Systemically injected exosomes targeted to EGFR deliver antitumor microRNA to breast cancer cells. Mol Ther 2013;21:185-91.

149 Antes TJ, Middleton RC, Luther KM, et al. Targeting extracellula vesicles to injured tissue using membrane cloaking and surface display. J Nanobiotechnology 2018;16:61.

150 Sterzenbach U, Putz U, Low L-H, et al. Engineered exosomes as vehicles for biologically active proteins. Mol Ther 2017;25:1269-78.

151 Sonoda T, Matsuzaki J, Yamamoto Y, et al. Serum microRNA-based risk prediction for stroke. Stroke 2019;50:1510-8.

152 Ophelders DRMG, Wolfs TGAM, Jellema RK, et al. Mesenchymal stromal cell-derived extracellular vesicles protect the fetal brain after hypoxia-ischemia. Stem Cells Transl Med 2016;5:754-63.

153 Xin H, Wang F, Li Y, et al. Secondary release of exosomes from astrocytes contributes to the increase in neural plasticity and improvement of functional recovery after stroke in rats treated with exosomes harvested from microRNA 133b-Overexpressing multipotent mesenchymal stromal cells. Cell Transplant 2017;26:243-57.

154 Moore TL, Bowley BGE, Pessina MA, et al. Mesenchymal derived exosomes enhance recovery of motor function in a monkey model of cortical injury. Restor Neurol Neurosci 2019;37:347-62.

155 Chen $\mathrm{K}-\mathrm{H}$, Chen $\mathrm{C}-\mathrm{H}$, Wallace CG, et al. Intravenous administration of xenogenic adipose-derived mesenchymal stem cells (ADMSC) and ADMSC-derived exosomes markedly reduced brain infarct volume and preserved neurological function in rat after acute ischemic stroke. Oncotarget 2016;7:74537-56.

156 Huang X, Ding J, Li Y, et al. Exosomes derived from PEDF modified adipose-derived mesenchymal stem cells ameliorate cerebral ischemia-reperfusion injury by regulation of autophagy and apoptosis. Exp Cell Res 2018;371:269-77.

157 Zheng Y, He R, Wang P, et al. Exosomes from LPS-stimulated macrophages induce neuroprotection and functional improvement after ischemic stroke by modulating microglial polarization. Biomater Sci 2019;7:2037-49. 